\title{
Hypothesis: Obesity Is Associated with a Lower Mutation Threshold in Colon Cancer
}

\author{
Michael Bordonaro, Darina Lazarova ${ }^{凶}$ \\ Department of Basic Sciences, The Commonwealth Medical College, 525, Pine Street, Rm. 3042, Scranton, PA 18509, USA
}

$\square$ Corresponding author: Darina Lazarova, PhD, Associate Professor of Molecular Biology, Department of Basic Sciences, The Commonwealth Medical College, 525 Pine Street, Scranton, PA, USA, email: dlazarova@tcmc.edu, tel. 570-504-9645, fax 570-504-9660

(c) 2015 Ivyspring International Publisher. Reproduction is permitted for personal, noncommercial use, provided that the article is in whole, unmodified, and properly cited. See http:/ /ivyspring.com/terms for terms and conditions.

Received: 2015.04.08; Accepted: 2015.06.12; Published: 2015.07.15

\begin{abstract}
Neoplastic progression requires accumulation of several mutations (mutation threshold). We hypothesize that obesity raises the risk of microsatellite stable (MSS) colon cancer (CC) at least in part by decreasing the mutation threshold. Thus, we posit that obese patients require fewer mutations, particularly driver mutations, compared to their normal BMI counterparts. Further, we suggest that the reduced number of required mutations in obese patients could be due to several factors, including the high levels of cytokines that accompany obesity. Cytokine-activated ERK, AKT, and JAK/STAT signaling could synergize with CC-initiating mutations to promote intestinal neoplastic development. Therefore, driver mutations that induce these specific pathways may not be "required" for neoplastic development in obesity; alteration in cell signaling consequent to obesity can substitute for some driver mutations in neoplastic progression. This hypothesis is supported by preliminary analyses of data from The Cancer Genome Atlas (TCGA). Thus, we observed that, compared to normal weight patients, cancer genomes of obese MSS CC patients exhibit fewer somatic mutations, and correspondingly lower numbers of mutations in driver genes $(P=0.026)$. The most striking observation was the lower number of KRAS mutations detected in patients with high body-mass index (BMI). These intriguing observations require further validation with increased number of patients, taking into account all possible confounding factors. If the hypothesis is confirmed, future studies should also address several possible explanations for the observed lower mutation threshold in obese MSS CC patients.
\end{abstract}

Key words: Colon cancer, obesity, mutations, BMI, cell signaling

\section{Introduction}

Obesity is a worldwide epidemic, and contributes to at least $11 \%$ of colon cancer (CC) cases [1,2]. Studies have shown an association between obesity and increased risk of CC [3-6]. We posited that, compared to CC patients with normal BMI (18.5-24.9), CC patients with $\mathrm{BMI} \geq 25$ exhibit a lower number of driver gene mutations. This hypothesis is supported by findings in a Drosophila model, in which tumor development was promoted in a set of cells with a Ras ${ }^{\mathrm{V} 12}$ mutation by cells secreting Jnk-induced cytokines [7]. In obesity, JNK signaling-induced cytokines are secreted by adipocytes and macrophages [8]; therefore, we hypothesized that cytokine-induced survival pathways cooperate with CC-initiating mutations to accelerate neoplastic development. Thus, according to our hypothesis, obesity induces physiological changes, possibly including alterations in cell signaling, which can substitute for some driver mutations that are typically observed as part of colonic neoplastic progression.

\section{Results and Discussion}

Although thorough evaluation of our hypothesis requires larger-scale studies with much larger data 
sets and more detailed analyses, we conducted small-scale preliminary analyses in order to evaluate our hypothesis in a currently existing data set.

Thus, we utilized whole exome sequencing and clinical data from The Cancer Genome Atlas (TCGA) (https://tcga-data.nci.nih.gov/tcga/, accessed on 08/21/2014). Statistical differences between group means were determined by one-way analysis-of-variance (ANOVA) and Tukey's multiple comparison test to adjust for multiple comparisons with 95\% confidence (GraphPad Prism 6 software). $P$-values lower than 0.05 were considered statistically significant.

From the database of 446 colon adenocarcinoma patients we obtained clinical and sequencing information for 175 patients; thus, body weight and height data were not available for more than half of the patients. The greatest number of somatic mutations was detected in patients with normal BMI: the median numbers of mutations for normal, overweight, and obese patients were 176.0, 123.0, and 129.5, respectively (dataset A, Table I). The large standard deviations in the mean number of mutations were due to microsatellite instability (MSI) cases. Unlike microsatellite stable (MSS) CCs, MSI tumors have a distinct mechanism of development [9]; therefore, we performed separate analyses of cases with $<500$ somatic mutations $(n=136)$ and cases with $\geq 500$ somatic mutations $(n=39)$. The cut-off value of 500 mutations was selected based upon the report by Vogelstein et al. [10], in which MSI colorectal cancers were categorized as those having more than 500 nonsynonymous mutations. The analyses of the 136 MSS CCs (dataset B, Table I) confirmed that increasing BMI was associated with a decreasing number of mutations, although these differences were not statistically significant. Possible confounding factors were the inclusion of silent and passenger mutations, and the pathology stage of the neoplasms. Therefore, our next step was to analyze the number of mutations in driver genes only. Our criteria for driver genes were based upon published data (ref.10, supplementary tables S2A and S2B), and the CC mutations reported by the sequencing project of Broad Institute of Harvard and MIT (http://www.tumorportal.org/). Based upon these analyses, patients with normal BMI exhibited an average of $5.3 \pm 2.4$ mutations in driver genes, overweight patients exhibited $4.6 \pm 1.9$, and obese patients had the least number of mutations of $4.1 \pm 1.8$ (dataset D, Table I). There was a statistically significant difference in the number of mutations in driver genes between the three groups of patients as determined by one-way ANOVA, $F(2,133)=3.47, P=0.034$. Tukey's post-hoc test revealed that the statistically significant difference was in the number of mutations in driver genes between normal weight and obese patients, $P=$ 0.026. In this cohort of 136 patients, the normal BMI patients were staged as follows: T1 (1), T2 (5), T3 (26), $\mathrm{T} 4$ (1), T4a (5), and T4b (2); the 53 overweight patients were staged at T1 (2), T2 (4), T3 (42), T4a (3), and T4b (2), and the obese patients were staged at Tis (1), T2 (9), T3 (32) and T4a (1). Considering that more advanced cancers exhibit a greater number of somatic mutations and vice versa, we re-analyzed the data for MSS CCs staged at T3, since these cases constituted the majority of all cases. Among the T3-stage CCs, one-way ANOVA established statistically significant differences, $F(2,97)=4.327, P=0.016$. The Tukey's post-hoc test confirmed that there was a statistically significant difference in the number of mutations in driver genes between normal weight and obese patients $(5.5 \pm 2.4$ versus $3.9 \pm 1.8, P=0.012)$, but not between normal weight and overweight patients (5.5 \pm 2.4 versus $4.5 \pm 2.0$ ), or between overweight and obese patients. The 39 MSI CCs were analyzed separately, and the number of mutations in driver genes did not decrease with increase in BMI (dataset E, Table I).

The three most frequently mutated genes in MSS CCs were APC, TP53, and KRAS; whereas, in MSI CCs the most frequently mutated genes were $A R, R N F 43$, and BRAF (Table II). Significantly, among the MSS CCs, the number of KRAS mutations was lower in patients with higher BMI: normal weight patients exhibited a KRAS mutation frequency of $55.0 \%$, whereas overweight and obese patients exhibited $47.2 \%$ and $32.6 \%$, respectively. Mutations in BRAF, $N R A F$, or HRAS did not compensate for the differences in the number of KRAS mutations (Table IIA). Another difference was that the tumor suppressor gene PTEN was mutated more frequently in normal weight patients with MSS and MSI CCs. As expected, the most prevalent mutations in MSS CC cases were these in APC, a gene encoding a regulator of WNT/beta-catenin activity, and normal weight, overweight, and obese patients exhibited $75.0 \%$, $81.4 \%$, and $80 \%$ APC mutation frequency (Table IIA). Two other genes involved in the regulation of WNT signaling, SOX9 and FAM123B (Table IIA), also exhibited slightly increased mutation frequency in overweight and/or obese patients, thus raising the possibility that patients with higher BMI develop colonic neoplasms with higher WNT activity levels.

To validate our results on frequency of genetic alterations, we utilized the cBioPortal for Cancer Genomics that provides analysis of large-scale cancer genomics data sets [11]. According to the cBioPortal for Cancer Genomics, in the 2012 Nature report on TCGA data, out of 212 colorectal cancer samples with copy number alterations and sequencing data, 162 
(76.4\%) of the cases exhibited altered APC gene, and $90(42.5 \%)$ exhibited altered KRAS gene [12]. These reported frequencies of $A P C$ and KRAS gene alterations are similar to the obtained by us estimates after combining the MSI and MSS CC cases in our datasets $(n=175)$; thus, in our analyses the frequency of genetic alterations in APC was $72.0 \%$, and in KRAS - $42.3 \%$. The slight differences in the gene frequency alterations likely due to the fact that all reported analyses combine colon and rectal adenocarcinoma cases; whereas, our analyses of TCGA data were focused only on colon adenocarcinomas.

Table I. Datasets utilized in the analyses. A. All CC cases with available TCGA data on body height, weight, and mutations based upon whole exome sequencing (as of August 2014). B. CC cases with available BMI data, and with less than 500 somatic mutations (including silent mutations that constitute approximately $30 \%$ of all mutations). C. CC cases with available BMI data and with more than 500 somatic mutations. $\mathbf{D}$ and $\mathbf{E}$. Numbers of mutations in driver genes in datasets $B$ and $C$. The asterisk in dataset $D$ denotes a statistically significant difference between the number of driver gene mutations in obese versus normal weight CC patients, $P=0.026$.

\begin{tabular}{|c|c|c|c|c|c|c|c|}
\hline \multicolumn{8}{|c|}{ A. ALL COLON ADENOCARCINOMA CASES $(\mathrm{n}=175)$} \\
\hline \multirow[t]{2}{*}{ Mean BMI } & \multirow{2}{*}{$\begin{array}{l}\text { Number of pa- } \\
\text { tients }\end{array}$} & \multicolumn{3}{|c|}{ Age of diagnosis } & \multicolumn{3}{|c|}{ Somatic mutations } \\
\hline & & Average & SD & Median & Average & SD & Median \\
\hline Normal 22.2 \pm 1.7 & 58 & 66.4 & 14.3 & 68.0 & 618.3 & 1062.9 & 176.0 \\
\hline Overweight $27.4 \pm 1.4$ & 61 & 63.5 & 14.0 & 65.0 & 264.0 & 394.0 & 123.0 \\
\hline Obese $35.1 \pm 5.2$ & 56 & 65.5 & 11.1 & 67.0 & 576.7 & 1232.4 & 129.5 \\
\hline \multicolumn{8}{|c|}{ B. MSS COLON ADENOCARCINOMA CASES, SOMATIC MUTATIONS $(n=136)$} \\
\hline \multirow[t]{2}{*}{ Mean BMI } & \multirow{2}{*}{$\begin{array}{l}\text { Number of pa- } \\
\text { tients }\end{array}$} & \multicolumn{3}{|c|}{ Age of diagnosis } & \multicolumn{3}{|c|}{ Somatic mutations } \\
\hline & & Average & SD & Median & Average & SD & Median \\
\hline Normal $22.0 \pm 1.7$ & 40 & 67.5 & 13.1 & 69.5 & 147.9 & 72.7 & 122.5 \\
\hline Overweight $27.3 \pm 1.4$ & 53 & 62.9 & 13.9 & 65.0 & 123.0 & 53.9 & 118.0 \\
\hline Obese $35.2 \pm 4.9$ & 43 & 65.7 & 10.2 & 67.0 & 124.3 & 57.7 & 116.0 \\
\hline \multicolumn{8}{|c|}{ C. MSI COLON ADENOCARCINOMA CASES, SOMATIC MUTATIONS $(n=39)$} \\
\hline \multirow[t]{2}{*}{ Mean BMI } & \multirow{2}{*}{$\begin{array}{l}\text { Number of pa- } \\
\text { tients }\end{array}$} & \multicolumn{3}{|c|}{ Age of diagnosis } & Somatic $\mathrm{m}$ & & \\
\hline & & Average & $\mathrm{SD}$ & Median & Average & SD & Median \\
\hline Normal 22.6 \pm 1.7 & 19 & 62.7 & 17.2 & 66.0 & 1621.5 & 1419.6 & 1257.0 \\
\hline Overweight $28.0 \pm 1.1$ & 7 & 68.6 & 15.8 & 73.0 & 1305.1 & 286.7 & 1279.0 \\
\hline Obese $32.9 \pm 3.3$ & 13 & 64.6 & 14.1 & 66.0 & 2072.9 & 1948.0 & 1463.0 \\
\hline LON ADF & & 10 & 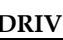 & $\operatorname{NES}(n=$ & & & \\
\hline Mean BMI & Number of patie & Mut & iverg & & & & \\
\hline & & Ave & & & SD & & \\
\hline Normal $22.2 \pm 1.7$ & 40 & 5.3 & & & 2.4 & & \\
\hline Overweight $27.4 \pm 1.4$ & 53 & 4.6 & & & 1.9 & & \\
\hline Obese $35.2 \pm 5.4$ & 43 & $4.1^{*}$ & & & 1.8 & & \\
\hline E. MSI COLON ADEI & ARCINOMA CA & , MUTAT & RI & $E S(n=39$ & & & \\
\hline Mean BMI & Number of patie & Mut & iver g & & & & \\
\hline & & Ave & & & SD & & \\
\hline Normal & 19 & 20.9 & & & 19.3 & & \\
\hline Overweight & 7 & 20.1 & & & 4.1 & & \\
\hline Obese & 13 & 22.9 & & & 10.9 & & \\
\hline
\end{tabular}

Table II. A. Mutations in driver genes of $136 \mathrm{CC}$ patients with less than 500 somatic mutations per neoplasm (dataset B, Table I). B. Mutations in driver genes of $39 \mathrm{CC}$ patients with more than 500 somatic mutations per neoplasm (dataset C, Table I).

\begin{tabular}{|c|c|c|c|}
\hline \multicolumn{4}{|c|}{ A. MSS Colon Adenocarcinoma Cases, $\mathrm{n}=136$} \\
\hline Driver gene & Normal $(n=40)$ & Overweight $(\mathrm{n}=53)$ & Obese $(n=43)$ \\
\hline AKT1 & 1 & 1 & 0 \\
\hline APC & 30 & 44 & 35 \\
\hline ATM & 2 & 4 & 1 \\
\hline ATRX & 4 & 0 & 1 \\
\hline$\overline{A R}$ & 8 & 7 & 8 \\
\hline ARID1A & 1 & 2 & 0 \\
\hline ARID2 & 0 & 1 & 1 \\
\hline ASXL1 & 1 & 1 & 1 \\
\hline$B 2 M$ & 0 & 2 & 0 \\
\hline BCLAF1 & 1 & 0 & 1 \\
\hline BRAF & 3 & 2 & 4 \\
\hline$B C O R$ & 1 & 3 & 0 \\
\hline BRCA1 & 0 & 0 & 1 \\
\hline BRCA2 & 1 & 3 & 1 \\
\hline CARD11 & 0 & 0 & 1 \\
\hline
\end{tabular}




\begin{tabular}{|c|c|c|c|}
\hline CASP8 & 0 & 1 & 0 \\
\hline CBL & 0 & 0 & 2 \\
\hline CCDC27 & 1 & 1 & 0 \\
\hline CDC73 & 2 & 1 & 1 \\
\hline CDKN2A & 1 & 0 & 0 \\
\hline CNBD1 & 0 & 1 & 0 \\
\hline CREBBP & 2 & 2 & 0 \\
\hline CTNNB1 & 2 & 3 & 1 \\
\hline DNMT3A & 0 & 2 & 1 \\
\hline ELF3 & 0 & 2 & 2 \\
\hline EP300 & 1 & 2 & 1 \\
\hline ERBB2 & 0 & 2 & 0 \\
\hline ERBB3 & 1 & 0 & 1 \\
\hline FAM123B & 1 & 8 & 0 \\
\hline FBXW7 & 4 & 4 & 5 \\
\hline FGFR3 & 1 & 0 & 0 \\
\hline FUBP1 & 0 & 0 & 2 \\
\hline GATA3 & 1 & 0 & 0 \\
\hline GNAQ & 1 & 0 & 0 \\
\hline GNAS & 1 & 1 & 2 \\
\hline GOT1 & 0 & 0 & 1 \\
\hline HRAS & 1 & 0 & 1 \\
\hline IDH1 & 0 & 2 & 0 \\
\hline IKZF1 & 1 & 1 & 0 \\
\hline JAK2 & 2 & 0 & 0 \\
\hline JAK3 & 0 & 1 & 1 \\
\hline KDM5C & 0 & 1 & 1 \\
\hline KDM6A & 2 & 1 & 0 \\
\hline KIAA1804 & 1 & 1 & 1 \\
\hline KIT & 0 & 4 & 0 \\
\hline KLF4 & 1 & 0 & 0 \\
\hline KRAS & 22 & 25 & 14 \\
\hline MAP2K4 & 2 & 1 & 2 \\
\hline МАРЗК10 & 0 & 1 & 0 \\
\hline MED12 & 2 & 1 & 0 \\
\hline MET & 1 & 0 & 0 \\
\hline MLL2 & 4 & 0 & 0 \\
\hline MLL3 & 3 & 4 & 1 \\
\hline MLH1 & 1 & 1 & 0 \\
\hline MYCL1 & 2 & 0 & 0 \\
\hline NCOA3 & 1 & 0 & 1 \\
\hline NCOR1 & 1 & 0 & 1 \\
\hline NF1 & 1 & 0 & 0 \\
\hline NOTCH1 & 0 & 1 & 1 \\
\hline $\mathrm{NOTCH} 2$ & 0 & 0 & 1 \\
\hline NRAS & 1 & 0 & 4 \\
\hline PAX5 & 1 & 0 & 0 \\
\hline PBRM1 & 4 & 1 & 0 \\
\hline PDGFRA & 1 & 0 & 2 \\
\hline PIK3R1 & 2 & 3 & 0 \\
\hline PRDM1 & 0 & 1 & 0 \\
\hline PTCH1 & 1 & 0 & 0 \\
\hline PTEN & 4 & 1 & 0 \\
\hline PTPN11 & 1 & 1 & 0 \\
\hline RB1 & 1 & 1 & 0 \\
\hline RBM10 & 0 & 1 & 2 \\
\hline RET & 1 & 1 & 2 \\
\hline RNF43 & 1 & 1 & 1 \\
\hline SETD2 & 0 & 1 & 3 \\
\hline SF3B & 2 & 0 & 0 \\
\hline SMAD2 & 1 & 0 & 1 \\
\hline SMAD4 & 5 & 7 & 6 \\
\hline SMARCA4 & 1 & 0 & 1 \\
\hline$S M O$ & 1 & 1 & 1 \\
\hline SOX9 & 3 & 7 & 5 \\
\hline STAG2 & 0 & 1 & 1 \\
\hline TCF7L2 & 3 & 5 & 0 \\
\hline TP53 & 24 & 35 & 27 \\
\hline TSHR & 1 & 1 & 0 \\
\hline \multicolumn{4}{|c|}{ B. MSI Colon Adenocarcinoma Cases, $n=39$} \\
\hline Driver gene & Normal $(n=19)$ & Overweight $(\mathrm{n}=7)$ & Obese $(\mathrm{n}=13)$ \\
\hline$A B L 1$ & 2 & 0 & 0 \\
\hline$A C V R 1 B$ & 2 & 1 & 2 \\
\hline AKT1 & 1 & 1 & 1 \\
\hline
\end{tabular}




\begin{tabular}{|c|c|c|c|}
\hline ALK & 3 & 1 & 2 \\
\hline APC & 8 & 1 & 8 \\
\hline$A R$ & 16 & 7 & 8 \\
\hline ARID1A & 9 & 2 & 6 \\
\hline ARID1B & 4 & 4 & 1 \\
\hline ARID2 & 3 & 3 & 2 \\
\hline ASXL1 & 3 & 1 & 2 \\
\hline ATM & 4 & 1 & 7 \\
\hline ATRX & 5 & 0 & 5 \\
\hline AXIN1 & 2 & 0 & 2 \\
\hline BAP1 & 0 & 0 & 1 \\
\hline$B 2 M$ & 4 & 1 & 1 \\
\hline BRAF & 8 & 5 & 6 \\
\hline$B C O R$ & 5 & 3 & 1 \\
\hline BRCA1 & 2 & 0 & 2 \\
\hline BRCA2 & 4 & 2 & 4 \\
\hline CARD11 & 1 & 1 & 5 \\
\hline CASP8 & 2 & 1 & 3 \\
\hline CCND1 & 3 & 0 & 0 \\
\hline CDC73 & 0 & 1 & 0 \\
\hline CDH1 & 5 & 2 & 1 \\
\hline CDKN2A & 1 & 0 & 1 \\
\hline CDKN2C & 0 & 0 & 2 \\
\hline $\mathrm{CIC}$ & 4 & 4 & 4 \\
\hline CRLF2 & 1 & 1 & 0 \\
\hline CSF1R & 3 & 1 & 0 \\
\hline CREBBP & 3 & 2 & 3 \\
\hline CTNNB1 & 5 & 2 & 1 \\
\hline CYLD & 1 & 0 & 0 \\
\hline DAXX & 2 & 1 & 0 \\
\hline DNMT1 & 1 & 0 & 1 \\
\hline DNMT3A & 1 & 2 & 1 \\
\hline EP300 & 1 & 0 & 2 \\
\hline ERBB2 & 1 & 1 & 1 \\
\hline EZH2 & 3 & 1 & 2 \\
\hline FAM123B & 3 & 0 & 0 \\
\hline$F B X W 7$ & 7 & 0 & 3 \\
\hline FGFR2 & 2 & 0 & 1 \\
\hline FGFR3 & 3 & 2 & 3 \\
\hline FLT3 & 1 & 0 & 0 \\
\hline FOXL2 & 1 & 0 & 4 \\
\hline FUBP1 & 3 & 0 & 0 \\
\hline GATA2 & 1 & 0 & 3 \\
\hline GATA3 & 2 & 0 & 2 \\
\hline GNA11 & 2 & 0 & 0 \\
\hline GNAQ & 1 & 0 & 2 \\
\hline GNAS & 2 & 1 & 4 \\
\hline HIST1H3B & 0 & 0 & 1 \\
\hline HNF1A & 2 & 2 & 3 \\
\hline IKZF1 & 0 & 1 & 0 \\
\hline JAK1 & 2 & 2 & 4 \\
\hline JAK2 & 2 & 0 & 1 \\
\hline JAK3 & 1 & 1 & 0 \\
\hline KDM5C & 3 & 0 & 0 \\
\hline KDM6A & 2 & 0 & 1 \\
\hline KIT & 4 & 2 & 0 \\
\hline KLF4 & 1 & 1 & 1 \\
\hline KRAS & 5 & 3 & 5 \\
\hline LMO1 & 0 & 0 & 1 \\
\hline MAP2K1 & 1 & 0 & 1 \\
\hline MAP2K4 & 1 & 1 & 2 \\
\hline МАРЗК10 & 1 & 2 & 2 \\
\hline MDM2 & 1 & 1 & 1 \\
\hline MED12 & 3 & 1 & 3 \\
\hline MEN1 & 3 & 0 & 1 \\
\hline$M E T$ & 1 & 0 & 3 \\
\hline MLL2 & 4 & 5 & 8 \\
\hline MLL3 & 8 & 3 & 6 \\
\hline MLH1 & 4 & 2 & 1 \\
\hline MSH2 & 2 & 1 & 3 \\
\hline MSH5 & 1 & 0 & 0 \\
\hline MSH6 & 4 & 1 & 2 \\
\hline$M Y C$ & 3 & 0 & 1 \\
\hline MYCL1 & 1 & 0 & 1 \\
\hline
\end{tabular}




\begin{tabular}{|c|c|c|c|}
\hline MYCN & 1 & 0 & 0 \\
\hline MYD88 & 1 & 0 & 0 \\
\hline NCOA3 & 3 & 1 & 1 \\
\hline NCOR1 & 7 & 0 & 4 \\
\hline NF1 & 7 & 3 & 5 \\
\hline NF2 & 3 & 0 & 0 \\
\hline NOTCH1 & 2 & 2 & 3 \\
\hline $\mathrm{NOTCH} 2$ & 3 & 0 & 2 \\
\hline NPM1 & 1 & 0 & 1 \\
\hline NKX2-1 & 0 & 1 & 1 \\
\hline NRAS & 0 & 1 & 0 \\
\hline PAX5 & 3 & 0 & 3 \\
\hline PBRM1 & 1 & 1 & 1 \\
\hline PDGFRA & 3 & 0 & 2 \\
\hline PIK3R1 & 5 & 1 & 1 \\
\hline PPP2R1A & 1 & 2 & 1 \\
\hline PRDM1 & 1 & 1 & 1 \\
\hline PTCH1 & 6 & 3 & 2 \\
\hline PTEN & 9 & 0 & 1 \\
\hline PTPN11 & 1 & 1 & 1 \\
\hline RB1 & 2 & 2 & 1 \\
\hline RET & 3 & 0 & 4 \\
\hline RNF43 & 8 & 6 & 7 \\
\hline SETBP1 & 3 & 3 & 4 \\
\hline SETD2 & 2 & 0 & 3 \\
\hline SF3B1 & 2 & 2 & 0 \\
\hline SMAD2 & 1 & 0 & 0 \\
\hline SMAD4 & 4 & 1 & 2 \\
\hline SMARCA4 & 3 & 2 & 3 \\
\hline SMARCB1 & 0 & 1 & 2 \\
\hline$S M O$ & 0 & 0 & 1 \\
\hline SOX9 & 2 & 1 & 3 \\
\hline SPOP & 1 & 0 & 0 \\
\hline TET2 & 3 & 3 & 2 \\
\hline TNFAIP3 & 4 & 1 & 1 \\
\hline TP53 & 4 & 1 & 4 \\
\hline TRAF7 & 0 & 0 & 1 \\
\hline TSC1 & 0 & 1 & 4 \\
\hline TSHR & 0 & 1 & 2 \\
\hline U2AF1 & 0 & 0 & 1 \\
\hline WT1 & 2 & 0 & 0 \\
\hline
\end{tabular}

Our preliminary observations suggest that in MSS CC, obesity associates with a lower number of mutations in driver genes; however, these results do not prove causality. There are several possible explanations for the observed association. One possibility is that obesity-associated cytokine-induced survival pathways (e.g., ERK and AKT) lower the mutation threshold for CC, since in obesity the activation of these pathways does not have to occur via driver gene mutations. Therefore, after MSS CC-initiating mutations (such as those in $A P C$ ), obesity-maintained survival signaling may promote neoplastic progression. Alternatively, obesity is characterized by a different colonic microbiome [13] that may affect the metabolic pathways and the levels of inflammatory molecules in the host. Another possibility is that in obese patients, epigenetic changes, rather than obesity-induced survival signaling, compensate for the lower number of mutations in driver genes. All of these possible explanations need to be explored in future molecular studies.

In our dataset of 136 patients with MSS CC, individuals with higher than normal BMI were diag- nosed at a younger age than patients with normal BMI (dataset B, Table I). Although these age differences were not statistically significant, the finding that excessive body weight associates with a lower mutation threshold may explain the trend of CC becoming a disease of younger individuals. Thus, colorectal cancer incidence has increased among 20- to 34-year olds in the past 35 years and, if the trend continues, there will be up to a $90 \%$ increase in CC incidence in this age group by 2030 [14]. This dramatic shift in age of diagnosis is likely due to the increasing obesity among children and adolescents [15]. Indeed, considering that CC progression takes 10 to 20 years, a simple calculation suggests that colon carcinogenesis may be initiated in elementary school- and middle school-age children.

In our analyses, we included the analyses of MSI CC samples as a negative control. Thus, MSI tumors constitute only $15 \%$ of all CC cases, exhibit a distinct mechanism of development [9], and whereas high BMI is associated with MSS colorectal cancer, it is not associated with MSI colorectal cancer [16]. Therefore, our conclusion that obesity is associated with a lower 
mutation threshold applies for the majority of the CC cases, which are of the MSS type; the findings reported here support our hypothesis specifically for MSS CC.

Limitations on our analyses were imposed by several factors. First, the sample size was relatively small due to the fact that sequencing is still expensive, and whole exome/genome sequencing and clinical data are not widely available. Second, TCGA's data do not provide information on factors that could confound the association between obesity and the number of mutations. For example, we utilized BMI recorded at the time of diagnosis; however, colonic carcinogenesis typically requires 10 to 20 years. It is therefore reasonable to consider the BMI history of the patient; however, such data are not collected by any institution that performs large scale genome sequencing. Third, the mutation data in TCGA are based upon whole exome sequencing and theoretically, mutations in non-coding regions could compensate for the lower number of mutations in obese patients. Therefore, proper validation or refutation of the hypothesis that obesity promotes MSS CC progression accompanied by fewer driver mutations will require, at minimum, larger data sets including more detailed patient information, including, but not limited to, BMI history of the patient.

If validated in future studies, our findings may have implications for both MSS CC prevention and treatment of individuals with excessive body weight. In terms of treatment, it is logical to posit that neoplasms with fewer driver gene mutations are more sensitive to molecularly targeted therapies. However, obesity-supported signaling may promote resistance to therapeutic agents, and therefore, should be taken into account when designing treatment strategies. In terms of prevention, body weight management is essential, and CC surveillance starting at a younger age should be considered for individuals with consistently higher than normal BMI.

\section{Abbreviations}

CC: colon cancer; TCGA: The Cancer Genome Atlas, MSS: microsatellite stable, MSI: microsatellite instability.

\section{Acknowledgements}

$\mathrm{DL}$ and $\mathrm{MB}$ are supported by funding of The Commonwealth Medical College.

\section{Competing Interests}

The authors declare no competing financial interests in relation to the reported analyses.

\section{References}

1. James PT, Leach R, Kalamara E, et al. The Worldwide Obesity Epidemic Obesity Res. 2001; 9: 228S-33S.

2. Wolin KY, Carson K, Colditz GA. Obesity and cancer. Oncologist 2010; 15: 556-65.

3. Limburg PJ, Anderson KE, Johnson TW, et al. Diabetes mellitus and subsite-specific colorectal cancer risks in the Iowa Women's Health Study. Cancer Epidemiol Biomarkers Prev. 2005; 14: 133-7.

4. Takahashi H, Yoneda K, Tomimoto A, et al. Life style-related diseases of the digestive system: colorectal cancer as a life style-related disease: from carcinogenesis to medical treatment. J Pharmacol Sci. 2007; 105: 129-32.

5. Aleksandrova K, Nimptsch K, Pischon T, et al. Obesity and colorectal cancer. Front Biosci. 2013; 5: 61-77.

6. Bardou, M., Barkun AN, Martel M. Obesity and colorectal cancer. Gut 2013; 62: 933-47.

7. Wu M, Pastor-Pareja JC, Xu T. Interaction between Ras(V12) and scribbled clones induces tumour growth and invasion. Nature 2010; 463: 545-8.

8. McArdle MA, Finucane OM, Connaughton RM et al. Mechanisms of obesity-induced inflammation and insulin resistance: insights into the emerging role of nutritional strategies. Front Endocrinol. (Lausanne) 2013; 4: 52.

9. Boland CR, Goel A. Microsatellite instability in colorectal cancer. Gastroenterology 2010; 138: 2073-87.e3.

10. Vogelstein B, Papadopoulos N, Velculescu VE, et al. Cancer genome landscapes. Science 2013; 339: 1546-58

11. [Internet] Center for Molecular Oncology and the Computational Biology Center at Memorial Sloan-Kettering Cancer Center. cBioPortal for Cancer Genomics. http://www.cbioportal.org/

12. Cancer Genome Atlas Network. Comprehensive molecular characterization of human colon and rectal cancer. Nature 2012; 487: 330-7.

13. Ley RE. Obesity and the human microbiome. Curr Opin Gastroenterol. 2010; 26: 5-11.

14. Bailey $\mathrm{CE}, \mathrm{Hu} \mathrm{CY}$, You YN, et al. Increasing Disparities in the Age-Related Incidences of Colon and Rectal Cancers in the United States, 1975-2010 JAMA Surg. 2015; 150: 17-22.

15. Ogden CL, Carroll MD, Kit BK, et al. Prevalence of childhood and adult obesity in the United States, 2011-2012. JAMA 2014; 311: 806-14.

16. Campbell PT, Jacobs ET, Ulrich CM, et al. Case-control study of overweight, obesity, and colorectal cancer risk, overall and by tumor microsatellite instability status. J Natl Cancer Inst. 2010; 102: 391-400. 